\title{
Development of a high-stability Microstrip-based L-band radiometer for ocean salinity measurements
}

\author{
Fernando A Pellerano, Kevin A. Horgan \\ Goddard Space Flight Center \\ Greenbelt, Maryland USA \\ Fernando.A.Pellerano@nasa.gov
}

\author{
William J Wilson, Alan B Tanner \\ Jet Propulsion Laboratory \\ California Institute of Technology \\ Pasadena, California USA
}

\begin{abstract}
The development of a microstrip-based L-band Dicke radiometer with the long-term stability required for future ocean salinity measurements to an accuracy of $0.1 \mathrm{psu}$ is presented. This measurement requires the $L$-band radiometers to have calibration stabilities of $\leq 0.05 \mathrm{~K}$ over 2 days. This research has focused on determining the optimum radiometer requirements and configuration to achieve this objective. System configuration and component performance have been evaluated with radiometer test beds at both JPL and GSFC. The GSFC testbed uses a cryogenic chamber that allows long-term characterization at radiometric temperatures in the range of $70-120 \mathrm{~K}$. The research has addressed several areas including component characterization as a function of temperature and DC bias, system linearity, optimum noise diode injection calibration, and precision temperature control of components. A breadboard radiometer, utilizing microstrip-based technologies, has been built to demonstrate this long-term stability.
\end{abstract}

Keywords-microwave radiometer; microstrip radiometer; noise injection radiometer; stability

\section{INTRODUCTION}

Aquarius is a new NASA Earth System Science Pathfinder (ESSP) mission to measure global sea surface salinity (SSS). Measurements of sea surface salinity (SSS) are important in understanding the response of the ocean to the global water cycle and climate changes. Accurate measurements of SSS, along with sea surface temperature, will determine the sea surface density, which controls the formation of water masses and regulates the 3-dimensional ocean circulation. The baseline science data product from the Aquarius mission will be a monthly global surface salinity map with $100-\mathrm{km}$ spatial resolution and with an accuracy of 0.2 practical salinity units over the 3-year mission life. (The practical salinity unit (psu) approximately corresponds to parts per thousand of salinity.) To achieve its objectives the calibration stability requirement for the Aquarius radiometers is $0.15 \mathrm{~K}$ over two days. Future SSS missions will require SSS measurements with an accuracy of $0.1 \mathrm{psu}$ and with a smaller spatial resolution. This will require the L-band radiometers to have calibration stabilities of $\leq 0.05 \mathrm{~K}$ over 2 days, or a factor of 3 improvement over the Aquarius radiometer requirement.

This research is part of NASA's Instrument Incubator Program (IIP) and is being conducted jointly between NASA's Goddard Space Flight Center (GSFC) and the Jet Propulsion

U.S. Government work not protected by U.S. Copyright
Laboratory (JPL). Prior work on this research had focused on thermal models of the radiometer electronics and on calibration schemes that will be needed to achieve the required noiseequivalent delta-T (NEDT) and were reported in [1]. The work presented here covers the development of a test bed suitable for analyzing long-term calibration stability, linearity concerns, and the design of a microstrip-based radiometer to achieve the stated performance.

\section{LONG-TERM STABILITY TESTBED}

Radiometer test beds have been assembled at both the JPL and the GSFC to test radiometer characteristics. The GSFC radiometer test bed was built with a cryogenic load and operated in a thermal vacuum chamber, as shown in the block diagram in Fig. 1. This setup is capable of simulating radiometer input temperatures in the range from $70 \mathrm{~K}-120 \mathrm{~K}$, which is the expected range over the open ocean over periods of months. In order to assess the long-term radiometer calibration stability the test bed must provide radiometric inputs more stable than the radiometer itself. For our case this implies knowledge of input radiometric temperature to $<0.05$ $\mathrm{K}$. To achieve this we apply both active control of the load and corrections based on a model for the input cable losses and emissions. This model breaks the cable into small sections and computes the cascaded system noise temperature based on the cable physical temperature distribution and includes the effects of connector losses and mismatches [2]. Fig. 2 shows a typical distribution of the sensors and the cable temperatures. The

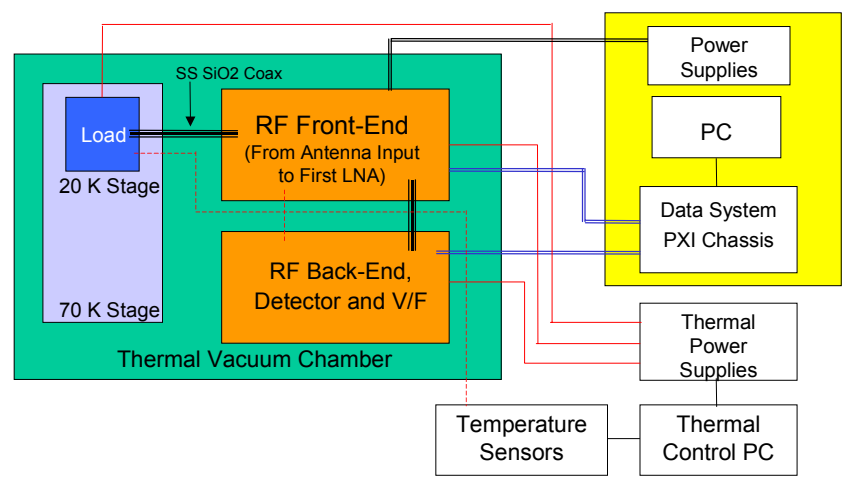

Figure 1. GSFC's Cryongenic Test Bed Block Diagram 


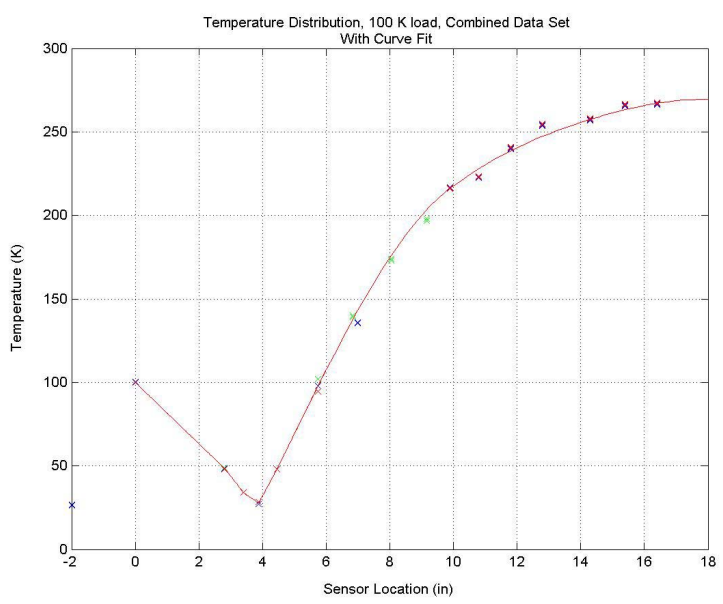

Figure 2. Temperature Distribution of the Coaxial Cable Between the Load and Radiometer Input. The sensor location 4 inches from the load represents a sink to the cryostat which isolates the load from changes on the warm side.

temperature control at the load is $<0.002 \mathrm{~K}$ and $\sim 0.1 \mathrm{~K}$ at the radiometer end of the cable. The coaxial cable is made of stainless steel with a silicon dioxide dielectric to provide good thermal isolation between the load and the radiometer. A sensitivity analysis to all parameters in the model suggested that the input temperature to the radiometer was stable with an uncertainty of $\sim 0.03 \mathrm{~K} \mathrm{RSS}$, which is adequate for the stability testing.

\section{RADIOMETER LINEARITY}

A constant deflection method was used to test and characterize the radiometer linearity. Using this approach, nonlinearities are observed as deviations of the noise diode deflection as the antenna noise temperature changes. This method offers the advantage that it can be applied to the complete radiometer system, as opposed to just the final detector circuit. In fact, this method can often be applied without any special accommodations or tests since the routine data from any noise adding radiometer may be sufficient to characterize the linearity of the system.
Fig. 3 shows the laboratory configuration of the deflection test. The antenna in this case was replaced with a cold source and an injected noise source, which combined could be adjusted between $\sim 30 \mathrm{~K}$ and $4700 \mathrm{~K}$, well below and above the expected operational range. Also, the noise diode was injected after the Dicke switch so that the deflection can be measured in both the 'antenna' and 'reference' modes of the switch. With both of these measurements we can normalize the antenna deflections and examine the linearity with the deflection ratio,

$$
D=\frac{V_{A N}-V_{A}}{V_{O N}-V_{O}}
$$

where the four voltages represent the response to the antenna $\left(V_{A}\right)$, antenna plus noise diode $\left(V_{A N}\right)$, ambient temperature reference $\left(V_{O}\right)$, and reference plus noise diode $\left(V_{O N}\right)$. In a linear system with no impedance mismatches $D$ should always be unity. If the system is not linear then $D$ will change as the antenna noise temperature changes.

As shown by the red ' + ' in Fig. 4, the system has a gain expansion behavior, as expected from the detectors, at low power levels up to approximately $2 \mathrm{~mW}$, and gain compression at the higher levels. The response can be linearized very successfully with an error $<0.04 \%$ using a third order polynomial fit, as shown by the blue ' $x$ '. These results also showed a bias in the deflection ratio due to the impedance mismatch of the Dicke switch between the antenna and load ports. This effect arises due to the coupler's imperfect isolation. Placing an isolator between the Dicke switch and the coupler eliminated this problem.

\section{MICROSTRIP DESIGN}

The results of device measurements previously made during this research [1] clearly demonstrate that thermal control of the radiometer is the single most dominant factor in obtaining long-term calibration stability. Corrections due to device temperature coefficients can be applied successfully up to a point. Time variable gradients, however, are significantly harder to correct. As a result we have determined thermal control of the radiometer to within $\pm 0.1 \mathrm{~K}$ is necessary to achieve the required calibration stability. A microstrip

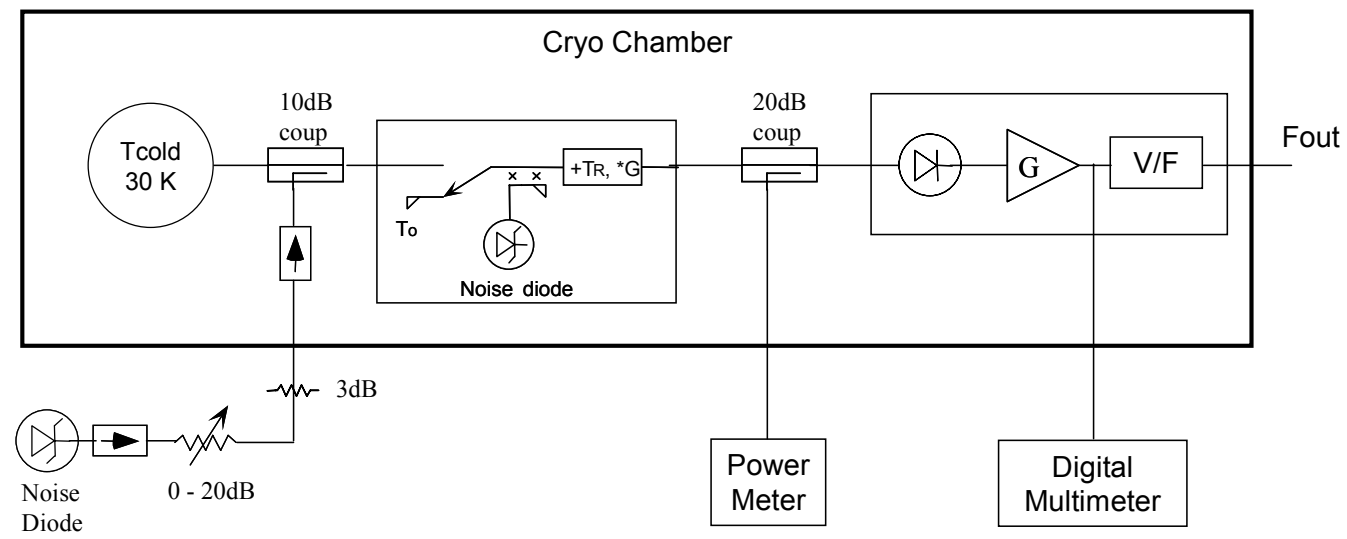

Figure 3. Linearity Test Configuration

U.S. Government work not protected by U.S. Copyright 


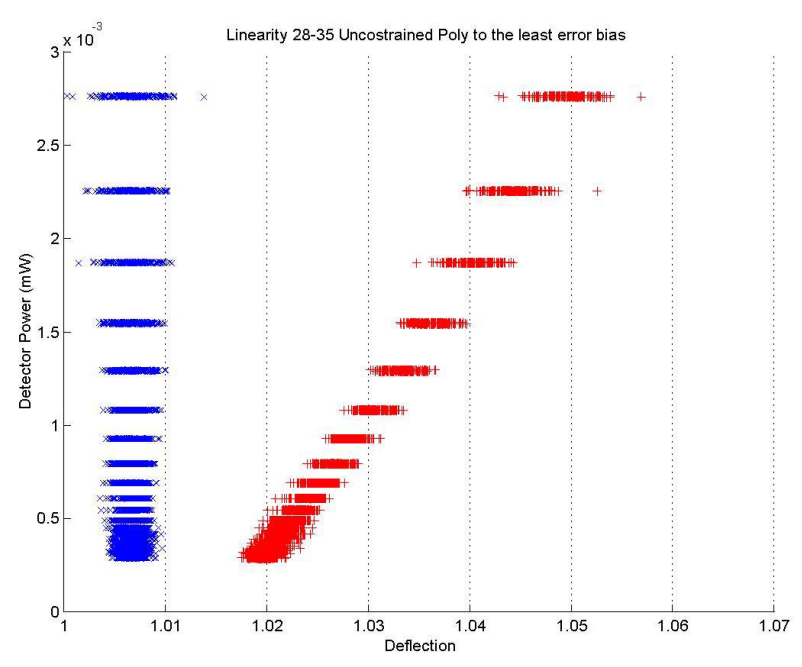

Figure 4. Corrected and Uncorrected Radiometer Deflections

implementation of the radiometer was developed to minimize the size and therefore improve thermal stability. This approach also allows every device and transmission line to be in intimate contact with a temperature controlled surface.

Fig. 5 shows a mechanical rendering of two sections of the radiometer. The housing design carefully considered the broadband performance of devices to avoid interference and instabilities for the active devices, like the low noise amplifiers. Circuit cavities behave like waveguides and must be designed with the appropriate cutoff frequency. In this design the cavities provide a margin of $>30 \mathrm{~dB}$ between gain stages. Additionally, filtered connectors are used at every DC or low frequency input/output (I/O) as well as internal connections. These radiometers will be completed by the summer of 2004 at which time their calibration stability will be assessed with the cryogenic test bed as described earlier.
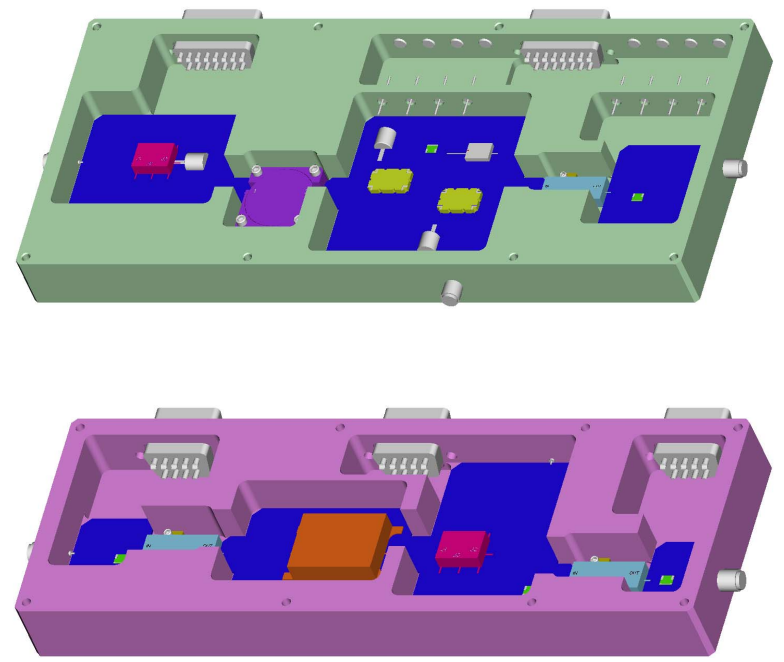

Figure 5. Mechanical Rendering of the Microstrip Radiometer

\section{ACKNOWLEDGMENT}

This work was carried out jointly between the Goddard Space Flight Center and the Jet Propulsion Laboratory, California Institute of Technology, under contract with the National Aeronautic Space Administration, as part of the Earth Science Technology Office Instrument Incubator Program.

\section{REFERENCES}

[1] Tanner, A., Wilson, W., Pellerano, F., "Development of a High Stability L-band Radiometer for Ocean Salinity Measurements", Proceedings of the 2003 International Geoscience and Remote Sensing Symposium, July21-25, 2003, Toulouse, France.

[2] F. T. Ulaby, R. K. Moore, and A. K. Fung, Microwave Remote Sensing: Active and Passive. Norwood, MA: Artech House, 1981, vol.1, p. 352.

U.S. Government work not protected by U.S. Copyright 